\title{
ON THE IDEALS OF STRICTLY SINGULAR AND INESSENTIAL OPERATORS ${ }^{1}$
}

\author{
WILLIAM PFAFFENBERGER
}

Abstract. In the present paper, we prove that if $X$ is a subprojective Banach space, then the ideal of strictly singular operators on $X$ is equal to the ideal of inessential operators on $X$. We give an example to show that equality does not hold for all Banach spaces $X$.

We also investigate the relationship between the semi-Fredholm operators on a Banach space and the right and left null divisors in the quotient algebra of all the bounded operators modulo the ideal of compact operators. We are able to get some complete characterizations of the null divisors when the Banach space is subprojective.

1. Introduction. If $X$ is a Banach space, let $B(X)$ denote the Banach algebra of all bounded linear operators from $X$ into itself. An operator $T \in B(X)$ is said to be strictly singular [4] if $T$ is not a homeomorphism when restricted to any closed infinite-dimensional subspace of $X$. Let $S(X)$ denote the closed two-sided ideal of strictly singular operators in $B(X)$. Let $\operatorname{Rad} A$ denote the Jacobson radical of any ring $A$. If $K(X)$ denotes the ideal of compact operators on $X$, then define $I(X)=\pi^{-1}[\operatorname{Rad}(B(X) / K(X))]$, where $\pi$ is the canonical homomorphism from $B(X)$ onto $B(X) / K(X) . I(X)$ is a closed two-sided ideal of $B(X)$, called the ideal of inessential operators. A Banach space $X$ is subprojective if, given any closed infinite-dimensional subspace $M$ of $X$, there exists a closed infinite-dimensional subspace $N$ contained in $M$ and a continuous projection of $X$ onto $N$. Subprojective spaces were investigated by $\mathrm{R}$. J. Whitley [8]. In $\$ 2$ we prove that if $X$ is subprojective, then $I(X)=S(X)$. We also give an example to show that equality does not always occur. In $\$ 3$ we investigate the relationship between the semi-Fredholm elements of $B(X)$ and the null divisors in $B(X) / K(X)$.

2. For any ring $A, x \in A$ is properly left quasi regular if $y x$ is left quasi regular for all $y \in A$. N. H. McCoy [6] characterized $\operatorname{Rad} A$ as the set of all $x \in A$ which are properly left quasi regular. Therefore

Received by the editors July 8, 1969 and, in revised form, January 12, 1970.

AMS Subject Classifications. Primary 4725, 4740.

Key Words and Phrases. Strictly singular operators, inessential operators, compact operators, Jacobson radical, subprojective Banach space, semi-Fredholm operators.

1 This work was partially supported by NSF Research Grant GP-8382 and is based on the author's doctoral dissertation at the University of Oregon. 
if $X$ is any Banach space and $T \in B(X)$, then $\pi(T) \in \operatorname{Rad}(B(X) / K(X))$ if and only if $\pi(T)$ is properly left quasi regular in $B(X) / K(X)$, but this is true if and only if $\pi(I)-\pi(W) \pi(T)$ is left invertible in $B(X) / K(X)$ for all $W \in B(X)$, where $I$ is the identity operator on $X$, or equivalently by $\left[9\right.$, p. 609] $I-W T \in \Phi_{+}(X)$ and there exists a continuous projection of $X$ onto the range of $I-W T$ for all $W \in B(X)$. Here $\Phi_{+}(X)$ are all operators in $B(X)$ with finite-dimensional null space and closed range. $\Phi(X)$ will denote the Fredholm operators in $B(X)$.

Lemma 2.1. Let $X$ be any Banach space. Then $T^{*} \in I\left(X^{*}\right)$ implies that $T \in I(X)$.

Proof. M. Schechter $[7,1143]$ remarks that $F(X)=\{T \in B(X) \mid$ $T+U \in \Phi(X)$ for all $U \in \Phi(X)\}$ is the largest ideal contained in the set of Riesz operators. This characterization also holds for $I(X)$ by [5], so $I(X)=F(X)$. Therefore suppose $T \notin I(X)$, then there exists a $U \in \Phi(X)$ such that $T+U \notin \Phi(X)$. B. Yood [9, p. 601] proved that $W \in \Phi(X)$ if and only if $W^{*} \in \Phi\left(X^{*}\right)$. So $T^{*}+U^{*} \notin \Phi\left(X^{*}\right)$ and $U \in \Phi(X)$ implies $U^{*} \in \Phi\left(X^{*}\right)$. Therefore $T^{*} \notin I\left(X^{*}\right)$ by the equivalence of $I\left(X^{*}\right)$ and $F\left(X^{*}\right)$. So $T^{*} \in I\left(X^{*}\right)$ implies $T \in I(X)$.

Theorem 2.2. Let $X$ be any subprojective Banach space. Then $S(X)=I(X)$.

Proof. S. R. Caradus $[1$, p. 66] proved that $S(X) \subset I(X)$ for any Banach space $X$.

To prove the converse take $T \in I(X)$ and suppose $T \notin S(X)$. Hence there exists a closed infinite-dimensional subspace $X_{1} \subset X$ such that $T$ is a homeomorphism of $X_{1}$ onto $T\left(X_{1}\right) . T\left(X_{1}\right)$ is a closed infinitedimensional subspace of $X$, so since $X$ is subprojective there exists an infinite-dimensional closed complemented subspace $X_{2} \subset T\left(X_{1}\right)$, where $X=X_{2} \oplus X_{3}$. Define $\tilde{T} \in B(X)$ by $\tilde{T} \equiv T^{-1}$ on $X_{2}$ and $\widetilde{T} \equiv 0$ on $X_{3}$. Since $T \in I(X)$ we know $\pi(T) \in \operatorname{Rad}(B(X) / K(X))$, so $I-W T$ $\in \Phi_{+}(X)$ for all $W \in B(X)$, in particular $I-\widetilde{T} T \in \Phi_{+}(X)$. This implies the null space of $I-\widetilde{T} T$ is finite-dimensional. $\widetilde{T}$ is a homeomorphism on $X_{2}$, so $\widetilde{T}\left(X_{2}\right)$ is an infinite-dimensional subspace of $X$ and $\tilde{T} T \widetilde{T}\left(X_{2}\right)$ $=\tilde{T}\left(X_{2}\right)$, since $T \tilde{T}$ is the identity on $X_{2}$. Therefore $(I-\tilde{T} T)\left(\tilde{T}\left(X_{2}\right)\right)$ $=0$, which implies $\widetilde{T}\left(X_{2}\right)$ is contained in the null space of $I-\widetilde{T} T$. This implies the null space of $I-\tilde{T} T$ is infinite-dimensional, a contradiction. Therefore $T \in S(X)$, so $I(X) \subset S(X)$.

An Example. I. A. Feldman, I. C. Gohberg and A. S. Markus [3] give an example of an operator, $V$, on $X=l_{q} \oplus L_{p}, 1<p<q<2$, such that $V \notin S(X)$ but $V^{*} \in S\left(X^{*}\right)$. This example shows that our theorem cannot be generalized to reflexive spaces (in fact $X$ is also 
superprojective [8, p. 255]), since $V^{*} \in S\left(X^{*}\right) \subset I\left(X^{*}\right),[1]$, implies $V \in I(X)$ by Lemma 2.1 , but $V \in S(X)$, so this implies $S(X) \neq I(X)$.

Note that this example also shows that a converse to $[8$, Theorem 2.2, p. 254] is not possible, since $X^{*}=l_{q^{\prime}} \oplus L_{p^{\prime}}$ with $2<q^{\prime}<p^{\prime}$ implies $X^{*}$ is subprojective, but $V^{*} \in S\left(X^{*}\right)$ and $V \equiv V^{* *} \in S\left(X^{* *}\right)$.

3. Let $N^{l}\left(N^{r}\right)$ denote the set of left (right) null divisors in $B(X) / K(X)$, and let $G^{l}\left(G^{r}\right)$ denote the set of left (right) invertible elements in $B(X) / K(X)$. For each $T \in B(X)$ let $N(T)$ denote the null space of $T$, and $R(T)$ denote the range of $T$. Let nul $T$ be the dimension of $N(T)$ and def $T$ be the codimension of the closure of $R(T)$ in $X$. Set

$$
\Phi_{-}(X)=\{T \in B(X) \mid \operatorname{def} T<\infty, \text { range } T \text { closed }\} .
$$

The members of $\Phi_{+}(X)$ and $\Phi_{-}(X)$ are called the semi-Fredholm elements of $B(X)$.

[ ] ${ }^{c}$ shall denote the set complementation.

B. Yood [9, p. 609] has shown that the following characterizations hold for any Banach space $X$ :

$$
\pi^{-1}(G)=\Phi(X),
$$

$\pi^{-1}\left(G^{r}\right)=\left\{T \in \Phi_{-}(X) \mid\right.$ there exists a continuous

projection onto $N(T)\}$,

and

$\pi^{-1}\left(G^{l}\right)=\left\{T \in \Phi_{+}(X) \mid\right.$ there exists a continuous

projection onto $R(T)\}$.

It follows trivially that:

and

$$
\begin{aligned}
\pi^{-1}\left(N^{l}\right) \cap \pi^{-1}\left(N^{r}\right) & \subset[\Phi(X)]^{c}, \\
\pi^{-1}\left(N^{l}\right) & \subset\left[\pi^{-1}\left(G^{l}\right)\right]^{c},
\end{aligned}
$$

$$
\pi^{-1}\left(N^{r}\right) \subset\left[\pi^{-1}\left(G^{r}\right)\right]^{c} .
$$

Lemma 3.1. Let $X$ be any Banach space. Then

$$
\pi^{-1}\left(N^{r}\right) \subset\left[\Phi_{-}(X)\right]^{c} .
$$

Proof. Suppose $T \in \pi^{-1}\left(N^{r}\right) \cap \Phi_{-}(X)$. Then $T \in \pi^{-1}\left(N^{r}\right)$ if and only if there exists a $Q \notin K(X) \ni Q T=W \in K(X)$, and $T \in \Phi_{-}(X)$ implies def $T<\infty$, so $X=R(T) \oplus M$, where $M$ is finite-dimensional.

Clearly $Q$ is not compact on $R(T)$, since $Q$ compact on $R(T)$ implies $Q$ compact on $X=R(T) \oplus M$, contrary to the choice of $Q$. But $\left.Q\right|_{R(T)}$ 
not compact implies $Q T \notin K(X)$, which is a contradiction, so $\pi^{-1}\left(N^{r}\right)$ $\cap \Phi_{-}(X)=\varnothing$ which implies $\pi^{-1}\left(N^{r}\right) \subset\left[\Phi_{-}(X)\right]^{c}$.

The following proposition is due to B. Yood [9, p. 600].

Proposition 3.2. Let $X$ be any Banach space. Then $T \in \Phi_{+}(X)$ if and only if given a bounded set $E$ in $X$ which is not totally bounded, then $T(E)$ is not totally bounded.

Let $S_{X}$ denote the closed unit ball of $X$.

Lemma 3.3. $\pi^{-1}\left(N^{l}\right) \subset\left[\Phi_{+}(X)\right]^{c}$.

Proof. Suppose $T \in \pi^{-1}\left(N^{l}\right) \cap \Phi_{+}(X)$, then $T \in \pi^{-1}\left(N^{l}\right)$ if and only if there exists a $Q \notin K(X)$ such that $T Q=W \in K(X)$. Also $T \in \Phi_{+}(X)$. $Q\left(S_{X}\right)$ is bounded but not totally bounded, so $T Q\left(S_{X}\right)$ is not totally bounded by Proposition 3.2. But this implies $T Q \notin K(X)$, a contradiction. So $\pi^{-1}\left(N^{l}\right) \cap \Phi_{+}(X)=\varnothing$ which implies $\pi^{-1}\left(N^{l}\right) \subset\left[\Phi_{+}(X)\right]^{c}$.

R. J. Whitley $[8$, p. 255] showed that the following Banach spaces are subprojective:

(i) $H$; any Hilbert space,

(ii) $l_{p}(1 \leqq p<\infty)$,

(iii) $c_{0}$,

(iv) $L_{p}(S, \Sigma, \mu)(2<p<\infty) ; S=[0,1],(\Sigma, \mu)$ Lebesgue measure.

Lemma 3.4. Let $X$ be subprojective. Then $T \notin \Phi_{+}(X)$ implies there exists an infinite-dimensional closed complemented subspace $X_{1} \subset X$ and a $K \in K(X)$ such that $T=K$ on $X_{1}$.

Proof. M. Schechter [7, p. 1142] proved that $T \notin \Phi_{+}(X)$ implies there exists a $K \in K(X) \operatorname{nul}(T-K)=\infty$, in other words $T=K$ on nul $(T-K)$, an infinite-dimensional closed subspace of $X$. Therefore since $X$ is subprojective there exists an infinite-dimensional closed complemented subspace $X_{1} \subset \operatorname{nul}(T-K)$. So $T=K$ on $X_{1}$, as desired.

Theorem 3.5. Let $X$ be subprojective. Then

$$
\pi^{-1}\left(N^{l}\right)=\left[\Phi_{+}(X)\right]^{c} .
$$

Proof. By Lemma 3.3, $\pi^{-1}\left(N^{l}\right) \subset\left[\Phi_{+}(X)\right]^{c}$. But if $T \notin \Phi_{+}(X)$, then by Lemma 3.4 , there exists an infinite-dimensional closed complemented subspace $X_{1} \subset X$ and a $K \in K(X)$ such that $T=K$ on $X_{1}$. Let $Q \in B(X)$ be the continuous projection of $X$ onto $X_{1} . Q \notin K(X)$, since if $Q \in K(X)$ then $R(Q)=X_{1}$ being closed would imply $R(Q)$ is finite-dimensional, which would be a contradiction.

Define $K_{1} \in K(X)$ by setting $K_{1} \equiv K$ on $X_{1}$ and $K_{1} \equiv 0$ on $X_{2}$, where $X=X_{1} \oplus X_{2} . T Q=K_{1} \in K(X)$ and $Q \notin K(X)$ implies that $\pi(T) \in N^{l}$, which implies $T \in \pi^{-1}\left(N^{l}\right)$. So $\left[\Phi_{+}(X)\right]^{c} \subset \pi^{-1}\left(N^{l}\right)$. 
Theorem 3.6. Let $X$ be a reflexive Banach space with $X^{*}$ subprojective. Then $\left[\Phi_{-}(X)\right]^{c}=\pi^{-1}\left(N^{r}\right)$.

Proof. By Lemma 3.1, $\pi^{-1}\left(N^{r}\right) \subset\left[\Phi_{-}(X)\right]^{c}$. Take $T \notin \Phi_{-}(X)$, then by $[9$, p. 601$], T^{*} \notin \Phi_{+}\left(X^{*}\right)$, so by Theorem 3.5 we have that $T^{*} \in \pi^{-1}\left(N^{l}\right.$, with respect to $\left.X^{*}\right)$, in other words there exists a $Q \notin K\left(X^{*}\right)$ and a $K \in K\left(X^{*}\right)$ such that $T^{*} Q=K$.

Let $\sigma$ be the natural isomorphism of $X$ into $X^{* *}$. $\sigma$ is onto since $X$ is reflexive and with this natural identification of $X$ and $X^{* *}$, we have $T^{* *}=T$. Since $\left(T^{*} Q\right)^{*}=K^{*}$ we have that $Q^{*} T^{* *}=K^{*} . K^{*} \in$ $K\left(X^{* *}\right)$ and $Q^{*} \notin K\left(X^{* *}\right)$ by Schauder's theorem [2, p. 485], so this implies that $T \in \pi^{-1}\left(N^{r}\right)$. Thus we have shown that $\left[\Phi_{-}(X)\right]^{c}$ $\subset \pi^{-1}\left(N^{r}\right)$.

Corollary 3.7. If $X=l_{p}(1<p<\infty)$ or any Hilbert space, then

(a) $\left[\Phi_{+}(X)\right]^{c}=\pi^{-1}\left(N^{l}\right)$,

(b) $\left[\Phi_{-}(X)\right]^{c}=\pi^{-1}\left(N^{r}\right)$, and

(c) $\left[\Phi_{+}(X) \cup \Phi_{-}(X)\right]^{c}=\pi^{-1}(N)$,

where $N=N^{l} \cap N^{r}$, the two-sided null divisors of $B(X) / K(X)$.

Proof. (a) follows from Theorem 3.5; (b) follows from Theorem 3.6; and (c) follows from the fact that $N=N^{\imath} \cap N^{r}$ implies

$$
\pi^{-1}(N)=\left[\Phi_{+}(X)\right]^{c} \cap\left[\Phi_{-}(X)\right]^{c}=\left[\Phi_{+}(X) \cup \Phi_{-}(X)\right]^{c} .
$$

\section{BIBLIOGRAPHY}

1. S. R. Caradus, Operators of Riesz type, Pacific J. Math. 18 (1966), 61-71. MR 33 \#7847.

2. N. Dunford and J. Schwartz, Linear operators. I: General theory, Pure and Appl. Math., vol. 7, Interscience, New York, 1958. MR 22 \#8302.

3. A. S. Markus, I. C. Gohberg and I. A. Feldman, Normally solvable operators and ideals associated with them, Bul. Akad. Štiince RSS Moldoven. 1960, no. 10 (76), 51-70. (Russian) MR 36 \#2004.

4. S. Goldberg, Unbounded linear operators: Theory and applications, McGrawHill, New York, 1966 MR 34 \#580.

5. D. Kleinecke, Almost-finite, compact, and inessential operators, Proc. Amer. Math. Soc. 14 (1963), 863-868. MR 27 \#5136.

6. N. H. McCoy, The theory of rings, Macmillan, New York, 1964. MR 32 \#680.

7. M. Schechter, Riesz operators and Fredholm perturbations, Bull. Amer. Math. Soc. 74 (1968), 1139-1144. MR 37 \#6777.

8. R. J. Whitley, Strictly singular operators and their conjugates, Trans. Amer. Math. Soc. 113 (1964), 252-261. MR 31 \#1565.

9. B. Yood, Properties of linear transformations preserved under addition of a completely continuous transformation, Duke Math. J. 18 (1951), 599-612. MR 13, 355.

University of Oregon, Eugene, Oregon 97403 and

University of Victoria, Victoria, B. C., CANADA 